\title{
Extract from Aronia melanocarpa fruits potentiates the inhibition of platelet aggregation in the presence of endothelial cells
}

Boguslawa Luzak', Jacek Golanski', Marek Rozalski², Urszula Krajewska², Beata Olas ${ }^{3}$, Cezary Watala ${ }^{1}$

1Department of Haemostasis and Haemostatic Disorders, Medical University Hospital No. 2, Medical University of Lodz, Poland

2Department of Pharmaceutical Biochemistry, Medical University of Lodz, Poland ${ }^{3}$ Department of General Biochemistry, University of Lodz, Poland

Submitted: 2 February 2009

Accepted: 23 March 2009

Arch Med Sci 2010; 6, 2: 141-144

DOI: 10.5114 /aoms.2010.13884

Copyright (c) 2010 Termedia \& Banach
Corresponding author: Prof. Cezary Watala, PhD Department of Haemostasis and Haemostatic Disorders Medical University Hospital No. 2

Medical University of Lodz 113 Zeromskiego St. 90-549 Lodz, Poland Phone: +48 426393471 Fax: +48 426787567 E-mail: cwatala@csk.umed.lodz.pl

\begin{abstract}
Introduction: Some polyphenolic compounds extracted from Aronia melanocarpa fruits (AM) have been reported to be cardioprotective agents. In this study we evaluated the ability of AM extract to increase the efficacy of human umbilical vein endothelial cells (HUVECs) to inhibit platelet functions in vitro.

Material and methods: This study encompasses two models of monitoring platelet reactivity: optical aggregation and platelet degranulation (monitored as the surface CD62P expression) in PRP upon the stimulation with ADP.

Results: We observed that only at low concentrations $(5 \mu \mathrm{g} / \mathrm{ml})$ did AM extract significantly improve antiplatelet action of HUVECs towards ADP-activated platelets in the aggregation test.

Conclusions: It is concluded that the potentiating effect of AM extract on the endothelial cell-mediated inhibition of platelet aggregation clearly depends on the used concentrations of Aronia-derived active compounds. Therefore, despite these encouraging preliminary outcomes on the beneficial effects of AM extract polyphenols, more profound dose-effect studies should certainly be considered before the implementation of Aronia-originating compounds in antiplatelet therapy and the prevention of cardiovascular diseases.
\end{abstract}

Key words: Aronia melanocarpa (chokeberry), endothelial cells, platelet aggregation, polyphenols, platelet reactivity.

\section{Introduction}

A substantial body of epidemiological literature suggests that the regular consumption of foods rich in polyphenols may reduce the risk of coronary heart disease and stroke [1]. In recent years a number of dietary sources inhibiting platelet function have been reported, but so far, due to the rather confusing array of conflicting evidence, the exact relationship between diet and platelet function remains unresolved [2]. There are no literature reports on the modulation of endothelial cell (EC)-induced inhibition of platelet aggregation by polyphenolic compounds. The general aim of this work is to evaluate the ability of the extract from Aronia melanocarpa fruits (AM) to improve EC efficacy at inhibiting platelet functions monitored under in vitro conditions. Berries of $A$. melanocarpa (chokeberries) have all the major healthy attributes and show the highest content of phenolic 
compounds (mainly anthocyanins) among the various natural products studied to date [3]. Special interest concerns significant protective action of chokeberry extracts/compounds on the cardiovascular system, and indeed, preliminary clinical studies have confirmed such compounds to be useful [4]. It has been reported that phenols (anthocyanins) from the chokeberry decreased blood pressure, lowered plasma lipid concentration and peroxidation, and reduced blood platelet reactivity, which makes them potentially interesting pharmaceuticals for cardiovascular therapy $[5,6]$. In our studies we observed that chokeberry extract may increase the antiplatelet action of human umbilical vein endothelial cells (HUVECs) after stimulation with ADP, and it is important to emphasize the significance of the dose-effect relationship of such interactions. We also tested some potential molecular mechanisms possibly determining such effects. However, further investigations are needed to fully explore the molecular mechanism(s) of such an alleviating influence of AM extract.

\section{Material and methods}

\section{Reagents}

The extract of $A$. melanocarpa was supplied by Agropharm SA (Poland). This extract contains ca. $60 \%$ total polyphenols, including a minimum of $20 \%$ anthocyanins. ADP was from Chrono-Log (Havertown, PA USA). Reagents for flow cytometry studies and VacutainerTM containing $0.105 \mathrm{M}$ buffered sodium citrate were from Becton Dickinson (San Diego, CA, USA). 6-keto-prostaglandin $F_{1 \alpha}$ EIA Kit was from Cayman Chemical Company (Ann Arbour, MI, USA).

\section{Blood collection}

Blood was collected from 16 healthy donors ( 8 men and 8 women, with a mean age of 29.8 \pm 12.4 year) into a vacuum tube containing $0.105 \mathrm{M}$ buffered sodium citrate. The blood was centrifuged for $6 \mathrm{~min}$ at $1100 \mathrm{rpm}$ to obtain the platelet-rich plasma (PRP). The platelet count in PRP was adjusted to $2 \times 10^{8}$ platelets $/ \mathrm{ml}$ prior to use in all experiments.

The study was performed under the guidelines of the Helsinki Declaration for human research and approved by the committee on the Ethics of Research in Human Experimentation at the Medical University of Lodz (No. RNN/13/07/KB).

\section{Cell cultures}

Human umbilical vein endothelial cells and all reagents needed for cell culture were purchased from Cascade Biologics (Portland, Oregon, USA). The HUVECs were cultured according to the manufacturer's instructions and the cells underwent 2-10 passages. In platelet aggregation studies, the HUVECs were suspended in Medium 200 at decreasing cell counts and finally in PRP the amount of HUVECs was $9.9 \times 10^{3}$ cells $/ \mathrm{ml}$ or $6.6 \times 10^{3}$ cells $/ \mathrm{ml}$ or $3.3 \times 10^{3}$ cells $/ \mathrm{ml}$. In flow cytometry studies the HUVECs were first transferred to 24-well plates at the count of $75 \times 10^{3}$ cells per well and these cells were further grown in a humidified atmosphere of $5 \% \mathrm{CO}_{2}$ in air at $37^{\circ} \mathrm{C}$ for $24 \mathrm{~h}$.

\section{Measurements of platelet reactivity}

\section{Aggregation}

Platelet-rich plasma was incubated $(10 \mathrm{~min}$, $\left.37^{\circ} \mathrm{C}\right)$ with $\operatorname{AM}(5,10,30 \mu \mathrm{g} / \mathrm{ml})$ and/or HUVECS suspended in Medium 200 without supplements. As a control, Medium 200 was used. Platelet reactivity in the presence of $10 \mu \mathrm{M}$ ADP was monitored for 10 min using a method of optical aggregation and the aggregation curves were analysed using Platelet Aggregation Monitoring and Analysis (PAMA) software [7].

\section{Flow cytometry}

Platelets in PRP were incubated with HUVEC on 24-well cluster dishes $\left(10 \mathrm{~min}\right.$ at $\left.37^{\circ} \mathrm{C}\right)$. The incubation with $A$. melanocarpa extract (final concentrations $0,5,10,20,30 \mu \mathrm{g} / \mathrm{ml}, 10 \mathrm{~min}$ at $37^{\circ} \mathrm{C}$ ) followed two patterns: 1) AM was added to the HUVECs in Medium 200; after 10-min incubation the culture medium was removed from the wells, and PRP $\left(2 \times 10^{8}\right.$ platelets $\left./ \mathrm{ml}\right)$ was added (pre-incubation of HUVECs with AM); 2) AM and PRP were directly added to the HUVECs growing on the wells (without pre-incubation of HUVECs with AM). Optionally, $10 \mu \mathrm{M}$ ADP was added to the platelet suspensions in the wells with HUVECs. After incubation, PRP was aspirated from wells, mixed and immediately fixed with CellFix ( $2 \mathrm{~h}$ at room temperature or overnight at $4^{\circ} \mathrm{C}$ ). After that, platelet surface CD62P expression was analysed for 5000 platelets using BD LSR II System (Becton Dickinson, San Diego, CA, USA).

\section{Measurements of prostacyclin}

The concentrations of 6-keto-prostaglandin $F_{1 \alpha}$, a stable metabolite of prostacyclin (PGI), were monitored in the aliquots of plasma obtained after platelet aggregation induced with ADP (following sample centrifugation at 10,000 rpm, 6 min, stored at $-70^{\circ} \mathrm{C}$ until assayed) using EIA Kit.

\section{Statistical analysis}

Mean \pm SEM is given for all parameters. ShapiroWilk test was used to verify whether the data were normally distributed and Student's $t$-test for paired 
data was employed to estimate the significance of differences, with Bonferroni correction for multiple comparisons, where needed.

\section{Results}

In the last few years there has been an increasing interest in screening substances, especially natural products from plants, which may stimulate endothelial cells to produce antiplatelet modulators (prostacyclin [PGI] and nitric oxide $\left[\mathrm{NO}^{\bullet}\right]$ ). Relevant to the topic of our study are the reports indicating that indirect effects of flavonoids on platelet inhibition may be dependent on endothelial cells. In our present study we have attempted to assess whether natural chokeberry extract ( $A$. melanocarpa fruits) may further attenuate platelet reactivity in addition to the endothelial cell-mediated inhibition of platelet function. This study encompasses two simple models of monitoring platelet reactivity: platelet aggregation and platelet degranulation (monitored as the surface CD62P expression) in PRP upon the stimulation of platelets with exogenous ADP. We observed a significant reduction in ADP-induced platelet aggregation in the presence of HUVECs, an effect which depended on the cell count in the endothelial cell cultures (the rates of inhibition were $46.9 \pm 5.7 \%$ for $9.9 \times 10^{3}$ cells $/ \mathrm{ml}, 40.5 \pm 7.1 \%$ for $6.6 \times 10^{3}$ cells $/ \mathrm{ml}$ and $36.3 \pm 2.8 \%$ for $3.3 \times 10^{3}$ cells $/ \mathrm{ml}$, respectively $p<0.001$ or less). We also revealed a significant reduction in CD62P (P-selectin) expression on the surface of ADPinduced platelets after incubation with HUVECs (the expression was $40.7 \pm 3.5$ vs. $29.6 \pm 3.2 \%$ for control without EC and for platelets with EC respectively, $p<0.001)$. Thus, our observations are consistent with those of other studies reporting reduced blood platelet reactivity by endothelial cells $[8,9]$.

To further monitor the expected intensifying effect of the chokeberry extract on inhibiting platelet reactivity in the presence of HUVECs, we finally used the culture with $3.3 \times 10^{3}$ cells $/ \mathrm{ml}$ (the values of maximal aggregation were $49.8 \pm 1.9$ vs.
$32.0 \pm 2.3 \%$ for platelets without EC and for platelets with $\mathrm{EC}$ respectively, $p<0.001)$. For the first time, our study evaluated the in vitro ability of extract from $A$. melanocarpa berries to increase the efficacy of endothelial cells in inhibiting platelet function. We observed that AM significantly improved antiplatelet action of HUVECs in the aggregation test and this effect was evidently dependent on AM concentration: while significant for low concentrations $(5 \mu \mathrm{g} / \mathrm{ml})$, it vanished when they were higher (Table I). Likewise, AM at a concentration of $10 \mu \mathrm{g} / \mathrm{ml}$ showed a tendency to deepen the HUVECmediated inhibition of ADP-stimulated platelet degranulation (lowered the CD62P expression), an effect which however remained statistically insignificant (Table I). In our study we used ADP as a platelet agonist, which is known to activate both platelets (activation and aggregation) and endothelial cells via $\mathrm{P} 2 \mathrm{Y}$ receptors [10]. Wihlborg et al. showed that endothelial $\mathrm{P} 2 \mathrm{Y}$ receptors mediate vessel dilatation by the release of EDHF, NO ${ }^{\bullet}$ and prostaglandins [11]. These products may therefore contribute to decreased platelet reactivity. Some reports have revealed that polyphenols such as procyanidins and anthocyanins (this group of polyphenols is also present in $\mathrm{AM}$ fruit extract) can induce a $\mathrm{NO}^{\bullet}$-dependent relaxation of the vessel wall and that P2Y purinoreceptors could be involved in this relaxing effect [12].

The available literature provides a plethora of examples on how various experimental protocols employed for monitoring platelet function might affect how we assess the monitored effects of polyphenols [13]. Furthermore, we have to be aware of the dose-effect relationships when studying antiplatelet and/or cardioprotective effects of plant extracts. Our present study clearly shows that natural polyphenolic compounds prove effective only when employed at the optimal concentration, whereas such effects may not deepen, or may even be reversed, with increasing concentrations. The significance of the above factors should not be underestimated, all the more so considering the huge array of inconsistent data on various agents of plant origin.

Table I. The inhibition of ADP-induced platelet reactivity by Aronia melanocarpa extract (AM) in the presence of endothelial cells

\begin{tabular}{|lccc|}
\hline $\begin{array}{l}\text { Concentration of } \\
\text { AM }[\mu \mathrm{g} / \mathrm{ml}]\end{array}$ & Aggregation & $\begin{array}{c}\text { CD62P expression } \\
\text { (not pre-incubated with AM) }\end{array}$ & $\begin{array}{c}\text { CD62P expression } \\
\text { (pre-incubated with AM) }\end{array}$ \\
\hline 0 & $36.3 \pm 2.8(n=11)$ & $27.5 \pm 3.7(n=14)$ & $25.3 \pm 2.6(n=11)$ \\
\hline 5 & $43.3 \pm 3.6(n=10)^{*}$ & $24.9 \pm 6.1(n=10)$ & $24.9 \pm 3.3(n=10)$ \\
\hline 10 & $35.3 \pm 3.5(n=10)$ & $21.3 \pm 5.1(n=12)$ & $28.3 \pm 2.9(n=11)$ \\
\hline 20 & $36.0 \pm 4.7(n=7)$ & $24.2 \pm 4.9(n=12)$ & $26.2 \pm 3.8(n=11)$ \\
\hline 30 & $37.8 \pm 3.4(n=9)$ & $13.4 \pm 6.4(n=12)$ & $28.3 \pm 4.2(n=11)$ \\
\hline
\end{tabular}

The extent of the inhibition of platelet reactivity calculated vs ADP-induced aggregation or CD62P expression for ADP-activated samples. EC count was $3.3 \times 10^{3}$ cells $/ \mathrm{ml}$. Significance of differences estimated with paired Student's t-test with Bonferroni correction for multiple comparisons. $A M$ in $5 \mu \mathrm{g} / \mathrm{ml}$ concentration significantly reduced platelet aggregation in the presence of $E C\left({ }^{*} p<0.05\right)$ 
In our study we have observed a significantly increased concentration of 6-keto prostaglandin $\mathrm{F}_{10}$ (a stable metabolite of prostacyclin) in plasma after ADP-induced platelet aggregation in the presence of HUVECs $(400.2 \pm 94.4 \mathrm{pg} / \mathrm{ml}$ for PRP with EC vs. $55.1 \pm 22.1 \mathrm{pg} / \mathrm{ml}$ for PRP without EC, $p<0.05$ ). Nevertheless, the presence of AM extract did not significantly enhance the increase of 6-keto $\mathrm{PGF}_{1 \alpha}$ concentration. These data may suggest that the antiplatelet effect of EC in the presence of low concentrations of $A M$ is most likely not mediated by $P G I$ generation. Even though these results neither provide convincing evidence for revealing the mechanisms of an indirect antiplatelet action of $A M$, nor explore the mechanisms of $A M-E C$ interaction(s), they certainly do encourage further investigations of this problem.

In conclusion, the results of our work support the concept that modulation and improvement of endothelial function with preparations of herbal origin (food supplements) may be beneficial in hampering exaggerated blood platelet reactivity. Importantly, the potentiating effect of AM extract on the endothelial cell-mediated inhibition of platelet aggregation is clearly concentration-dependent. Hence despite these encouraging preliminary outcomes on beneficial effects of AM extract polyphenols, more profound dose-effect studies should certainly be considered before implementing Aronia-derived extracts in antiplatelet therapy and the prevention of cardiovascular diseases.

\section{Acknowledgments}

This study was supported by project N405 065034 of the Polish Ministry of Science and Higher Education, grant 502-16-651 from the Medical University of Lodz.

\section{References}

1. Vita JA. Polyphenols and cardiovascular disease: effects on endothelial and platelet function. Am J Clin Nutr 2005; 81: 292S-7S.

2. Janaszewska A, Golanski J, Watala C. Natural compounds as modulators of platelet function. In: The activity of natural compounds in diseases prevention and therapy. Durackova Z. (ed); Rozum a Zdravie, o.z; Slovak Academic Press, 2007; 1: 199-225.

3. Kulling SE, Rawel HM. Chokeberry (Aronia melanocarpa): A review on the characteristic components and potential health effects. Planta Med 2008; 74: 1625-34.

4. Kowalczyk E, Krzesinski P, Fijalkowski P, Blaszczyk J, Kowalski J. The use of anthocyanins in the treatment of cardiovascular diseases [Polish]. Pol Merkur Lekarski 2005; 19: 108-10.

5. Broncel M, Kozirog-Kolacinska M, Andryskowski G, et al. Effect of anthocyanins from Aronia melanocarpa on blood pressure, concentration of endothelin-1 and lipids in patients with metabolic syndrome [Polish]. Pol Merkur Lekarski 2007; 23: 116-9.
6. Valcheva-Kuzmanova S, Kuzmanov K, Mihova V, Krasnaliev I, Borisova P, Belcheva A. Antihyperlipidemic effect of Aronia melanocarpa fruit juice in rats fed a highcholesterol diet. Plant Foods Hum Nutr 2007; 62: 19-24.

7. Golanski J, Golanski R, Iwaszkiewicz A, Chlopicki S, Gresner P, Watala C. Resistance to aspirin in patients after coronary artery bypass grafting is transient. Impact on the monitoring of aspirin antiplatelet therapy. Therap Drug Monit 2005; 27: 484-90.

8. Broekman MJ, Eiroa AM, Marcus AJ. Inhibition of human platelet reactivity by endothelium-derived relaxing factor from human umbilical vein endothelial cells in suspension: blockade of aggregation and secretion by an aspirin-insensitive mechanism. Blood 1991; 78: 1033-40.

9. Nosaka S, Nakayama K, Hashimoto M, et al. Inhibition of platelet aggregation by endocardial endothelial cells. Life Sci 1996; 59: 559-64.

10. Andre P, Delaney SM, LaRocca T, et al. P2Y12 regulates platelet adhesion/activation, thrombus growth, and thrombus stability in injured arteries. J Clin Invest 2003; 112: 398-406.

11. Wihlborg AK, Malmsjo M, Eyjolfsson A, Gustafsson R, Jacobson K, Erlinge D. Extracellular nucleotides induce vasodilatation in human arteries via prostaglandins, nitric oxide and endothelium-derived hyperpolarising factor. $\mathrm{Br}$ J Pharmacol 2003; 138: 1451-8.

12. Mendes A, Desgranges C, Cheze C, Vercauteren J, Freslon J. Vasorelaxant effects of grape polyphenols in rat isolated aorta. Possible involvement of a purinergic pathway. Fundam Clin Pharmacol 2003; 17: 673-81.

13. Nardini M, Natella F, Scaccini C. Role of dietary polyphenols in platelet aggregation. A review of the supplementation studies. Platelets 2007; 18: 224-43. 